WIENER SLAVISTISCHES JAHRBUCH, Band 54/2008, 47-62

(C) 2008 by Österreichische Akademie der Wissenschaften, Wien

EVA HAUSB ACHER

\title{
Poetik der Migration. Transnationale Literatur zeitgenössischer russischer und kroatischer Autoren
}

Autoren und Autorinnen der Migration vollziehen immer wieder einen realen Kulturwechsel zwischen ethnischen und nationalen semantischen Räumen. Ihre Biographien können als transnational bezeichnet werden und erzeugen hybride Räume zwischen den Kulturen. In ihren Texten werden oftmals diese kulturellen Hybridisierungsprozesse deutlich: Einerseits wird ihre literarische Gebundenheit an ,imaginäre Heimatländer", ihre symbolische Verortung in Raum und Zeit, Landschaften, Traditionen und Mythen offensichtlich. Gleichzeitig wird aber auch die Loslösung von dieser heimatbezogenen Verortung und mitgebrachten Identität virulent.

An anderer Stelle ${ }^{1}$ wurde von mir diese biographisch bedingte interkulturelle Konstellation am Beispiel russischer Migrationsautoren untersucht, und zwar nicht nur als Gegenstand der Texte, sondern auch in ihren narratologischen Konsequenzen. Welche konkreten literarischen Formen, Verfahren und narratologischen Strategien, von der Entscheidung für bestimmte Gattungen über die Favorisierung spezieller Themen, Motive und Topoi bis zum Gebrauch besonderer rhetorischer Mittel kennzeichnen eine Poetik der Migration? Am Beispiel Marija Rybakovas („Anna Grom i ee prizrak“, 1999) wurde die topographische Akzentuierung, die Thematisierung von Mobilität und die besondere Gestaltung literarischer Räume und Erinnerungslandschaften in der Migrationsliteratur beschrieben. In Julia Kissinas Textsammlung „Vergiß Tarantino“ (2005) werden die Textstrategien der Mimikry und Groteske offensichtlich, zwei stilistische Posen bzw. Darstellungsmodi, die einer Poetik der Migration zugeschlagen werden können. Vladimir Kaminer zeigt mit seinen Bestsellern von „Russendisko“ (2000) bis „Karaoke“ (2005) im Spiel mit national-

1 Siehe dazu Hausbacher (2006). 
kulturellen Auto- und Heterosterotypen, dass deren Dekonstruktion auch jenseits von Interventionen auf der Ebene der Hochkultur erfolgen kann.

In diesem Beitrag wird am Beispiel Marina Palejs, die 1995 aus Russland in die Niederlande migriert, zum einen die liminale Perspektivierung vieler Migrationstexte und die Duplizierung sowie Hybridisierung von Räumen gezeigt, zum anderen auf die Gattungspräferenz vieler Migrationsautoren hingewiesen, die bei (Kurz-)Prosa und Essay liegt.

Eine weitere Hypothese unserer Auseinandersetzung mit Migrationsliteratur ist die Differenz zwischen Migrations- und Emigrationsparadigma. Die traditionelle Emigrationsliteratur ist primär durch zwei Faktoren gekennzeichnet: die politische Motiviertheit und die Unfreiwilligkeit des Kulturwechsels, beides zwar außerliterarische Kriterien, die aber nicht unerhebliche Auswirkungen auf die ästhetische Gestaltung der Texte haben. Bleibt die Emigrationsliteratur weitgehend im Opferdiskurs verhaftet $^{2}$ und strebt eine Angliederung an die kanonische Nationalliteratur an - sie orientiert sich weiterhin an der Herkunftsliteratur, während sich Migrationsliteratur an der Aufnahmegesellschaft orientiert (vgl. Rösch 2004: 90) -, so entwickelt die Migrationsliteratur neue, im Zeichen der Transkulturalität stehende ästhetische Paradigmen. Dieser Paradigmenwechsel wird auch in der Forschungslandschaft sichtbar, in der es vermehrt zu einer Abwendung von thematischen und inhaltlichen Untersuchungskriterien, wie sie noch für die Exil- bzw. Emigrationsliteraturforschung kennzeichnend waren, hin zu vornehmlich ästhetischen und poetologischen Fragestellungen kommt.

In Bezug auf diese Differenzierung ist Dubravka Ugrešić, die 1990 ihre ,jugoslawische“ Heimat verlassen hat und heute ebenfalls in den Niederlanden lebt, aus unserer Sicht mit ihren Romanen und Essaybänden (noch) im Diskurs der Emigration verhaftet, obwohl ihre postmoderne Schreibweise, die bei Paul Lützeler (Lützeler 2005) in Verbindung mit postkolonialen Positionen des Schreibens gebracht wird, viele Kennzeichen einer Poetik der Migration trägt.

Dass transkulturelle Literatur der Migration für die Entwicklung der Philologien nicht ohne Konsequenz bleiben kann, bewirkt sie doch zunehmend eine Infragestellung der auf Kanon und Differenz bauenden ,Zentren“ der einzelnen Nationalliteraturen, kann hier nur angedeutet werden. Auch das von Homi K. Bhabha entwickelte Modell einer neuen „Weltliteratur“, das eine Antwort auf diese Problematik gibt, weil diese ihrem Ursprung nach nicht mehr an einen Ort gebunden ist und sich zwischen den Kulturen bewegt, kann hier nicht im Detail erörtert werden. Allerdings liefert dieses „Weltliteratur-Modell“ auch Argumente für die in allen osteuropäischen Ländern nach 1990 wieder aufgeflammte Diskussion um die Einheit der Nationalliteratur.

${ }^{2}$ „Im Ausland verbreiteten viele Schriftsteller/innen der dritten Emigration weiter ihre Erfahrungen mit dem politischen System und dem Alltag in der Sowjetunion. [...] Emigrant/innen der dritten Welle verlassen Russland in ihren Werken relativ selten und scheinbar nicht gern." (Tichomirova 2000: 167 f.) 


\section{Grenzräume und liminale Perspektiven: Marina Palej}

„Ясно, что тема границ безгранична. И первый бессознательный импульс провоцирует сосредоточиться на границах именно неозязаемых, то есть самых существенных: между любовью и ненавистью [...], между бесчисленными >я< в личном сонме художника, между сном и так называемой явью, между отрезками времени [...]. Да в первую очередь о границах невидимых. И все же, все же ..." (Palej 2003: о. Р.)

So leitet die seit 1995 in den Niederlanden lebende russisch-jüdische Autorin Marina Palej ihren 2002 in der Zeitschrift „Lichtungen“ erstpublizierten Essay „AUS: GANG, -WEG“ ${ }^{3}$ ein, um - eben dennoch - gleich im nächsten Abschnitt konkrete, geographische, räumliche Grenzen und deren Überschreitung zu fokussieren. So als würden unsichtbare, metaphysische, oft auch bloß metaphorisch konstruierte Grenzen einerseits und sichtbare, ganz real bestehende Grenzen andererseits einander bedingen.

Geboren wurde Marina Palej als Tochter eines Ingenieurpaares und Enkeltochter von Czernowitzer Juden 1955 in Leningrad. Dort ist sie auch aufgewachsen und hat ein Medizinstudium absolviert. Sie arbeitete mehrere Jahre als Ärztin, bevor sie von 1985 bis 1991 ein Studium der Literaturkritik an der Moskauer Hochschule für Literatur aufgenommen hat. Seit 1987 wurden von ihr Prosa, Dramen und Drehbücher (zwei Verfilmungen in den USA) veröffentlicht, sowie ihre Übersetzungen aus dem Niederländischen, Englischen, Griechischen und Slowenischen. In Russland erschienen seit 1991 vier Bände mit Erzählungen und Romanen. Ihre Werke wurden in zwölf Sprachen übersetzt. Darüber hinaus arbeitet Palej auch in den Bereichen Fotographie, Design und Performance, die sie zu einem „Fototheater“ zu synthetisieren versucht (Palej 2005: o. P.). Seit 1995 lebt sie in den Niederlanden mit Reisen und wechselnden Zwischenaufenthalten all around the world auf Einladung von verschiedenen Stiftungen wie z. B. der Heinrich-Böll-Stiftung in Deutschland oder diversen Kulturinstitutionen. Ihre Kinder leben in Israel.

In dem bislang einzigen veröffentlichten Interview, erschienen 2005 in der Zeitschrift „Delo“ (Ljubljana), beschreibt Marina Palej diese ihre ,in-between“-Situation eigentlich sehr positiv als befreiend und ihre Kreativität stimulierend. In den Niederlanden lebt sie am Meer und fühlt dort erstmals das, was I. Brodskij als „the earth's width“ bezeichnet hat (vgl. Palej 2005: o. P.). Sie lebt dort in einer Ausländergemeinde als eine der wenigen Frauen, die kein Kopftuch trägt, sehr zurückgezogen, fast wie eine Einsiedlerin, eine einsame Inselbewohnerin. Diese Situation gibt ihr so Palej - jene schöpferische Freiheit, die sie braucht. Unter monokulturellen Bedingungen, führt Palej weiter aus, wäre ihr Leben künstlich abgeschnitten. Ihre Kind-

${ }^{3}$ Auf Russisch ist der Text 2003 unter dem Titel „Vychod“ in der Zeitschrift „Vestnik Evropy“ erschienen. 
heit hat Marina Palej in der finnischen Landschaft um Petersburg verbracht, ${ }^{4}$ erlebt aber bereits während ihrer Schulzeit in den 1970er und 1980er Jahren einen häufigen Orts- und Aufenthaltswechsel, weil die Mutter als Chemikerin in die diversen Industriegebiete der Sowjetunion von der kasachischen über die tschuvaschische bis in die ukrainische Republik versetzt wird. Und dieses nomadische Wanderleben setzt sich später auch außerhalb Russlands fort:

\begin{abstract}
„Я отчетливо вижу, что мои отроческие скитания - хочу того или нет - во многом повторили себя, начиная с моей жизни вне России. Только осуществился этот повтор уже словно бы на новом витке. Мои маршруты проходили уже не через республики Советского Союза, но через государства Европейского Союза: Швеция, Германия, Австрия, снова Германия, Бельгия, снова Швеция, затем Италия, Франция ... Географическая амплитуда нарастает: Соединенные Штаты Америки ... Утром Флорида, вечером - Сиэтл ... Калейдоскопически (уже не могу разделить их в своей памяти), - мелькают Айова, Вермонт, Массачусетс, Огайо, Иллинойс - and so on, and so forth - можно по алфавиту.“ (Palej 2005: о. Р.)
\end{abstract}

Diese ihre durch Bewegung, Weg und Distanzen gekennzeichnete nomadische Lebensform sei von großer impulsgebender Bedeutung für ihr Schaffen, betont Palej im Interview und vergleicht diese Position mit dem Blick auf die Welt, die man nur von außen, aus weiter Entfernung als Ganzes in den Blick bekommt:

„Дистанция - это главнейший компонент в технологии творчества - по крайней меpe, в моей. Я подразумеваю дистанцию (дорогу), которую познаешь на своей Шкуpe. [...] Живая уже десять лет на значительной дистанции к своей изначальной культуре (,дистанция“ - ключевое для меня слово: одна из моих книг так и называется (,Long Dinstance, или Славянский акцент'), я наконец-то эту культуру разглядела.“ (Palej 2005: o. P.)

Das Interview schließt mit der Darlegung eines Identitätsverständnisses, das Marina Palej neben der Favorisierung des Nomadismus in die Nähe postkolonialer Positionen stellt: Es ist dies eine offene Identitätskonzeption, die das spielerische Einnehmen und Ausprobieren verschiedener Ich-Rollen einschließt, ähnlich wie Marina Palej dies in ihren Fotoarbeiten umsetzt, für die sie großteils selbst Modell steht.

In der kulturwissenschaftlichen Theoriebildung der letzten Jahre kommt es zu einer zunehmenden Dynamisierung des Grenzkonzepts, die die Bewegtheit und Räumlichkeit von Grenze zum Thema macht. Es geht nicht in erster Linie um agg-

${ }^{4}$ Von diesen frühkindlichen Eindrücken, die geprägt sind von der finnischen Landschaft, lebt bis heute ihre künstlerische Kreativität, ähnlich wie auch bei Nabokov oder Mandel'štam die Liebe zu dieser Landschaft in ihre Poetik eingegangen ist (vgl. Palej 2005: o. P.). „Мое дошкольное детство - это Ингерманландия. Так издревле называлась территория (то шведская, то финская, то российская) между Финским заливом и южной частью Ладоги. Короткий вариант названия - Ингрия. Сосны, ели, озера, валуны ... Снега и льды ... Мхи ... Тишина ... Именно Ингерманландия я обязана всем, что чувствую, что умею в литературе.“ (Palej 2005: о. Р.) 
ressiv ausgetragene Konflikte und Machtkämpfe an Grenzen und um Territorien, sondern eher um ein spielerisches Erproben von Grenzbestimmungen und Grenzentwürfen, nach denen Subjekte und Kollektive sich ,in between“ formen und nicht mehr als Einheiten voneinander abgrenzen (vgl. Benthien - Krüger-Fürhoff 1999: 11). Grenzen werden nicht mehr als Linien der Konfrontation, sondern als „Orte wider die Verabsolutierung der einen wie der anderen Seite“ (Hohnsträter 1999: 240) gesehen. Eine liminale Grenzauffassung konzipiert Grenze als Kontinuum, die - metaphorisch auf die kulturelle Bedeutung angelegt - einen Ausweg aus polarisierenden Essentialismen bietet: damit können Differenzen gedacht werden, ohne sie an Hierarchien und Oppositionen zu binden. Wenngleich Räume grundsätzlich durch ihre Grenzen in ein Innen und Außen gespalten werden, kennt bereits die Alltagssprache den Begriff des Zwischenraums. Der Zwischenraum ist einerseits zu verstehen als ein am Rande gelegener Unbestimmtheitsraum, der andererseits Elemente des Innen und Außen in sich vereinigt und darum zwischen den beiden Polen vermitteln kann. Diese liminale Grenzkonzeption ${ }^{5}$ geht in Anlehnung an Lacan von der „Persistenz des Fremden im Eigenen (Gehrke 1999: 16) aus und wird verstanden als ein Heterotop (M. Foucault), als ein in-between-space (H. K. Bhabha), als eine Enklave, die im Bereich des Innen das fremde Außen verkörpert.

„Im Zwischenraum lösen sich die überkommenen polarisierenden Kategorien des (kolonialen) territorialen Nationalstaates auf und geben den Blick auf (diskursive) Praktiken einer Neubestimmung des Raums.“ (Assmann 2006: 151)

Die Grenze gewinnt also heute - zumindest in der aktuellen Theoriediskussion wird dies postuliert - zunehmend die Funktion eines Übergangsraums. Hybride Grenzräume (,borderlands“) erscheinen als das, was Homi Bhabha „third spaces“ oder „,in-beetween-spaces“ nennt, Mary Louise Pratt (1992) „Kontaktzonen“ oder Ulf Hannerz (1996) u. a. auch mit „Kreolisierung“ im Auge haben. Der Grenzstreifen hat für diese Theoretiker eine ethische und ästhetische Dimension, er ist zugleich Freiraum und Spielraum (Hohnsträter 1999: 242). In der „Praxis“ sind es vor allem

${ }^{5}$ Besonders hervor hebt Hohnsträter selbstredend die beiden „Klassiker“ des Liminalen: Den Ethnologen Victor Turner, der im Anschluss an Arnold van Gennepes Überlegungen zu den „Rites de Passages“ von 1909 Liminalität unter Bezug auf Rituale als den Zustand einer labilen Zwischenexistenz ,betwixt and between the positions assigned and arranged by law, custom, convention and ceremonial" (Turner 1967: 95) bestimmt hat. Turner begreift die Schwellenphase als Herbeiführung einer Krise, durch die kulturelle Spielräume für Experimente und Innovationen eröffnet werden; denn , in liminality, new ways of acting, new combinations of symbols, are tried out, to be discarded or accepted" (Turner 1967: 110). Ebenso erwähnt Hohnsträter die kultursemiotischen Studien Jurij Lotmans, die sich auf einen Grenzbegriff beziehen und Grenze als eine Zone „,kultureller Zweisprachigkeit“ (Hohnsträter 1999: 238) fassen. Auch in Homi Bhabhas postkolonialer „Location of culture" (1994) ist Liminalität ein wesentlicher Begriff. Die permanenten Hybridisierungsprozesse, in denen Kulturen von ihren Grenzen aus definiert werden, machen die Unterscheidung zwischen Innen und Außen einer Kultur zunehmend irrelevant. 
die kulturellen Produktionen von Migrant(inn)en, die solche Übergangs- und Zwischenräume besetzen, die die Durchlässigkeit und Fragwürdigkeit eines linearen Grenzverständnisses beschreiben und die Potential für multiple Formen der Selbstkonstruktion bieten. Migrationsbewegungen evozieren diese hybriden Zwischenräume geradezu und verankern Diskurse, die nicht länger in nationalen oder kulturellen Zentren, sondern an deren Peripherien angesiedelt sind.

So wie Marija Rybakova, Julia Kissina oder Vladimir Kaminer schreibt sich auch Marina Palej mit ihrer Migrationserfahrung auf ihre ganz spezifische Art und Weise in diesen Diskurs ein. Am Beispiel zweier Essays zeigen wir im Folgenden, wie Marina Palej vielfältige Dynamiken, Topiken und Metaphoriken des Liminalen aufgreift und ein transnationales Identitätskonzept andenkt. Ihre Texte weisen eine starke topographische Akzentuierung auf und - wie die folgenden Beispiele zeigen werden - sprengen die zeitlich lineare Anordnung zugunsten einer flächig-mosaikartigen Zusammenführung der Ereignisse. Palej setzt rhetorische Strategien von Hybridität ein, um so ihren Textraum als Denkraum für Ideen der Ambivalenz, der Unbestimmtheit und Offenheit zu entwerfen. Darüber hinaus handelt es sich bei essayistisch konzipierter ästhetischer Erfahrung um eine spezifische Variante liminaler Erfahrung. Die Gattung des Essay ${ }^{6}$ unterstützt die experimentierende Reflexion kultureller Differenz, um die es Palej hier geht: Beispielsweise indem sie Menschen und Orte als Doppelgänger-Existenzen entwirft oder das Ich in Form eines kindlichen Abzählreims als multiples beschreibt. Palej nutzt die dem Essay als kritischem und literarischem Diskurs inhärente Doppelstruktur: indem sie „über“ Grenzen spricht, redet und denkt sie notwendig auch über diese hinaus (vgl. Benthien - Krü-

${ }^{6}$ Ein historischer Abriss würde zeigen, dass der Essay immer schon bevorzugter Schauplatz kultureller Reflexion war. Der kritische „Möglichkeitssinn“ avanciert zur Wahrnehmungsform des Essays. Adorno spezifiziert diese experimentierende Methodik, indem er in seinem Aufsatz über den Essay von 1954 diesen als Form des mangelnden Ursprungs, der produktiven Standpunktlosigkeit und als Ausdrucksform der Nichtidentität bezeichnet. „Ein Mischprodukt, [das] dem Bewusstsein der Nichtidentität Rechnung trägt [...]; radikal im Nichtradikalismus, in der Enthaltung von aller Reduktion auf ein Prinzip, im Akzentuieren des Partiellen gegenüber der Totale, im Stückhaften. [...] Er revoltiert zumal gegen die seit Platon eingewurzelte Doktrin, das Wechselnde, Ephemere sei der Philosophie unwürdig. [...] Die Beziehung auf Erfahrung - [...] ihr verleiht der Essay soviel Substanz wie die herkömmliche Theorie den bloßen Kategorien [...] die Momente verflechten sich teppichhaft. [...]; für seine Affinität zur offenen geistigen Erfahrung hat er mit dem Mangel an jener Sicherheit zu zahlen; der Essay schüttelt die Illusion einer einfachen, im Grunde selber logischen Welt ab. [...] Seine Differenziertheit ist kein Zusatz sondern sein Medium. [...] Er denkt in Brüchen, so wie die Realität brüchig ist [...] Diskontinuität ist dem Essay wesentlich.“ (Adorno 1984: 9 ff.) In der Terminologie der Postcolonial Studies würde man u. a. von Zügen des Fremden und Hybriden sprechen, die dem Essay in kultur- wie auch literarhistorischer Hinsicht zugewiesen werden können. Gerade für diese produktive Kombinatorik des Heterogenen, wie sie in der frühen Moderne bereits anklang und wie sie sich im Migrationsdiskurs umsetzt, bietet der Essay das geeignete Medium. 
ger-Fürhoff 1999: 12). Sie erweitert die Texte um ein selbstreflexives Moment und greift immer wieder auf ihre eigene migratorische Erfahrungswelt zurück. Dass die Migrationserfahrungen allerdings auch Mechanismen der Re-Stabilisierung streng dichotomer Grenzziehung hervorrufen, verschweigt Palej nicht und bringt immer wieder zur Sprache, dass dieses Aushalten von kulturellen Differenzerfahrungen auch sehr schmerzhaft ist.

\section{„VYCHOD“}

Wenden wir uns auf dieser Basis dem ersten Palej-Essay zu, von dem einleitend schon die Rede war, und der teppichhaft-fragmentarisch in fünfzehn Abschnitten das Phänomen „Grenze“ reflektiert. Dabei sind es zwei Geschichten, die den Text tragen und die die Autorin erinnert: Einmal jene vom Großvater väterlicherseits, Schneider von Beruf, der im Sommer 1945, als er aus dem Krieg heim nach Czernowitz kam, eine im deutschen Solingen hergestellte Stahlschere mitbringt. Als der Vater zum Studium nach Leningrad geht, nimmt er diese Schere mit und lässt sie auch nicht zurück, als er vierzig Jahre später nach Bonn auswandert. „,[Н]ожницы вернулись к себе на родину." (Palej 2003: о. P.) An und für sich wäre diese Geschichte - obwohl eine Geschichte der Bewegung und des Grenzüberschreitens - gerade das Gegenteil des von mir oben beschriebenen liminalen Diskurses, zielt sie doch auf das Ankommen in der Heimat ab. Wäre da nicht die Erklärung dieser Geschichte durch die Erählerin/Autorin: Sie meint nämlich in einer Umkehrung der konventionellen Perspektive, dass es die Schere war, die zum auslösenden Gegenstand, zum „Beweggrund" in der Biographie des Vaters wurde:

„[...] именно ножницы и оказались тем пусковым предметом, которому была назначена роль истинного двигателя событий. Ножницам необходимо было вернуться в точку своего начала, и они, используя людей, провоцируя ситуации, вызывая >исторические события८, в конечном итоге это и сделали ..."(Palej 2003: о. Р.)

Der Perspektivenwechsel, den Palej hier einführt, löst die Binäropposition von Subjekt (Großvater) und Objekt (Schere), von Heimat (Czernowitz) und Fremde (Deutschland) ebenso wie feste Identitäten und Maßstäbe auf.

Die zweite tragende Geschichte des Essays ist die Erinnerung an eine Fahrt, die die Erzählerin/Autorin in einem eisig-kalten Leningrader Vorortzug im Dezember 1978 gemacht hat. Auf dieser Fahrt versucht sie, sich in die Lektüre des Romans „Die Gewinner“ von Julio Cortázar zu vertiefen; vergeblich allerdings, denn sie ist mit ihren Gedanken ständig bei einem befreundeten Ehepaar, das nach Israel auswandert und sich - um allen Beteiligten mögliche Unannehmlichkeiten zu ersparen - ganz ohne Abschied aus dem Staub macht. „Дети на даче“ (Palej 2003: о. Р.), sagt deren Großvater am Telefon, also dort, wo sich die Autorin gerade mit dem Zug durchbewegt. Dabei verschmilzt dieser Raum zu einem synkretistischen Gebilde: Die finnische Landschaft, Israel und das Jenseits treffen aufeinander. In ihrer Erinnerung entwirft die Autorin die finnische Landschaft in der Umgebung von Peters- 
burg, durch die der Zug fährt, nicht mehr nur als jene geliebte Landschaft ihrer Kindheit, sondern ruft auch die russische Kriegs- und Eroberungsgeschichte dieses Gebiets auf. Die russische Identität ihrer Heimatstadt gerät dabei ins Wanken, denn sie ist stärker an finnische denn an russische Landschaftsbilder gekoppelt, und sie zitiert aus einem Brief von Mandel'štam aus Frankreich: „Маленькая аномалия: тоску по родине я испытываю не о России, а о Финляндии“ (Palej 2003: о. Р.). Ganz wesentlich bei der Beschreibung dieser topographischen Überlagerungen ist die zeitliche Distanz, von der aus die Erzählerin/Autorin spricht: „А между тем, в вагоне происходит путешествие и во времени.“(Palej 2003: о. P.). Diese Distanz löst ein stark selbstreflexives Moment aus, welches in einer Spaltung ihrer Ich-Vorstellung mündet:

\begin{abstract}
„Мне сейчас странно вспоминать о той молодой женщине с книгой. Что общего между нами? Какие-то паспортные данные ... Нет, даже они во многом изменились. Общее прошлое? Тоже нет: у той женщины >прошлогох было гораздо, гораздо меньше. [...] Где же граница? Возможно, кривой линией она проходит именно через подобные эпизоды. [...] Этот посыл напоминает мне знаменитый фильм Хичкока: арестовывается некий опасный двойник, который в финале, при ближайшем рассмотрении, не имеет ровным счетом ничего схожего с оригиналом.“ (Palej 2003: о. Р.)
\end{abstract}

In diese beiden „Haupt-Geschichten“ mischt Palej auch subjektiv erlebte „Mikroereignisse“, die sie in einer „Großaufnahme“ gleichberechtigt in ihren Erinnerungsstrom einreiht und so ihren Textteppich webt. An einer Stelle vergleicht sie Grenzüberschreitung mit einem Einweihungsritual: „[...] главный дар от Господа Бога - обновление всех чувств - вот что такое >переход границыґ.“ (Palej 2003: o. P.) Immer wieder streut sie aber auch ihre Bewegungs- und Übertretungseuphorie relativierende Bilder ein, beispielsweise das tragische Ereignis vom Tod von achtundfünfzig Chinesen, die im Juni 2000 in einem LKW von einer kriminellen Schlepperbande unter menschenunwürdigen Umständen transportiert wurden und am Hafen der Stadt Dover vor den Toren Englands zu Tode kamen.

Abschließend erzählt sie, wovon nun der Roman „Die Gewinner“ eigentlich handelt: Auf einem Schiff auf Weltreise befinden sich lauter Glückspilze, die eine Kreuzfahrt gewonnen haben. Die Besonderheit des Schiffes ist die, dass es keinen Zugang zum Heck gibt. Was steckt hinter dem Verbot, das Heck zu betreten? Einige der Passagiere wollen das Geheimnis lüften, übertreten damit die Grenzen des Erlaubten und gehen dafür sogar in den Tod. „И не только в книге.“ (Palej 2003: о. P.) - schließt der Text und markiert damit neben den in diesem Roman vermischten horizontalen und vertikalen, sichtbaren und unsichtbaren Grenzen auch den Übergang zwischen fiktiver und nicht-fiktiver Welt.

Insgesamt ist der Text also ein Plädoyer für ein vagabundierendes Grenzüberschreiten, für eine liminale Perspektivierung ihrer literarischen Grenzreflexionen. Als Gewährsleute für einen derartigen Lebens- und Schreibstil greift sie immer wieder auf den diasporischen Diskurs der russischen Literaturgeschichte zurück, in dem 
sie V. Nabokov, I. Bunin, O. Mandel'štam oder I. Brodskij zu Wort kommen lässt und ihren Text so stark intertextuell auflädt.

\section{„Graz: Ein Unterhaltsames Hologramm“"7}

Den Essay „Graz: Ein unterhaltsames Hologramm“ hat Marina Palej für die Anthologie „graz von aussen“ (2003) geschrieben, während sie zur Konzipierung des Programms „Graz - Europäische Kulturhauptstadt 2003“ nach Graz eingeladen war: „Zwei Monate Nirwana auf Dienstreise“ (Palej 2003a: 224), wie sie ihren Aufenthalt dort kurz zusammenfasst. Gleich anfänglich problematisiert sie ihre Situation als außen stehende Beobachterin, und vergleicht sie mit der eines Ethnographen, der seinem „Untersuchungsobjekt“ mittels „dichter Beschreibung“ gerecht zu werden versucht: „Wie die Stadt (das Außen), trennen von sich selbst (dem Innen)?“ (Palej 2003a: 219)

Mit dem Untertitel des zweiten Abschnittes „Eine neue Erde, aber kein neuer Himmel" markiert Marina Palej die hier entwickelte liminale Perspektive, die ihre Annäherung an die Stadt Graz ausmacht: Der Stadtraum Graz wird als Doppelgänger zum Czernowitz ihrer Kindheit entworfen, sie spricht von einer „Doppelgängerexistenz von Graz und Czernowitz“ (Palej 2003a: 222). Besonders in diesem Kapitel wird gut sichtbar, dass der Stadttext Graz als Traumsignatur lesbar wird. Das Wechseln der Orte geht fließend, grenzenlos vor sich, ohne die Distanz zwischen den Destinationen zu bemerken. Neben Czernowitz mischt sich immer wieder Petersburg in ihr Grazbild:

„Es waren eindeutig Petersburger Häuser, man braucht nicht einmal die Augen zusammenkneifen, um sie einzurahmen und deutlicher von der nicht-Petersburger Landschaft abzugrenzen, sie standen unerschütterlich im Einklang mit der selbstgenügsamen Logik meines Traumes: es gab gewissermaßen eine Verschiebung der Traumbilder [...].“ (Palej 2003a: 230)

Neben diesen Doppel- oder Mischorten werden auch sog. Mischpersonen entworfen, wie sie Sigmund Freud auch in der Traumarbeit ${ }^{8}$ aufgrund der Mechanismen der Verschiebung und Verdichtung beschreibt:

„Wenn man lange lebt, wird jede neue Bekanntschaft zu einer Kombination der Menschen, denen man früher einmal begegnet ist - jeder Ort setzt sich zusammen aus der Summe der Orte, die jemandes Seele passiert haben.“(Palej 2003a: 222)

Selbstreflexiv und unter Zuhilfenahme intertextueller Bezüge - auch hier sprechen wieder I. Brodskij, I. Bunin, V. Nabokov, Il' f und Petrov mit - problematisiert

${ }^{7}$ Der Text ist auf Russisch nicht publiziert, weshalb die Zitate auf Deutsch angeführt sind; das russische Manuskript trägt den Titel „Zanimatel'naja Golografija. Grac: vzgljad izvne" und ist wesentlich umfangreicher.

8 Dies führt Freud im sechsten Kapitel der 1900 erschienenen „Traumdeutung“ aus. 
sie die Unmöglichkeit, über Graz außerhalb fertiger Stereotype und klischeehafter Formeln zu sprechen. Gleichzeitig eröffnet sie mit dieser Erkenntnis aber auch ihr Spiel mit diesen Klischees und Bildern als mimikrierende Strategie. Ein Beispiel dafür bietet jene Textstelle, wo sie ein und dasselbe Bild zwei Mal hintereinander zeichnet, „einmal aus dem Blickwinkel einer Russin, ein zweites Mal aus dem einer frisch bekehrten Holländerin.“ (Palej 2003a: 225 f.)

„Rekognoszierung der Orte und Nationalitäten“ nennt sie das dritte Kapitel, in dem sie die Frage der nationalen Identität behandelt: sie gießt diese in die Form des oben schon angesprochenen Abzählreims und gibt ihr damit jenen spielerischen Freiraum für eine Konzeption des Transnationalen:

„Hätte man mir diese Frage gestellt, wer ich sei, aufgewachsen weit entfernt von den wolkenlosen Feldern der Toleranz (und des Grundsatzes, unter allen Umständen einen Konsens zu finden), wie würde ich antworten? So natürlich: mit Russen fühle ich mich als Jüdin, mit Juden natürlich als Russin, mit Europäern als Asiatin, und mit Asiaten als völlig Ausgestoßene. Auch ein Abzählreim.“(Palej 2003a: 224)

Interessant in unserem Zusammenhang ist auch die Differenzierung zwischen Emigration und Migration, die Marina Palej hier explizit ausführt: Beschreibt sie die Emigration in die Niederlande, ,wo man alles Nationale und Individuelle töten muss und nur die völlige Gesichtslosigkeit die Integration ermöglicht“ (Palej 2003a: 226) als negative Erfahrung, so sieht sie ihre migratorische Existenz in Graz, wo die „Vielseitigkeit der kulturellen Einflüsse mit Israel vergleichbar ist“ (Palej 2003a: 227) als Bereicherung: „In die Niederlande bin ich durch den Fleischwolf der Emigration gegangen, während ich in Graz einfach so lebte, ohne mich um den Rest der Welt zu kümmern.“ (Palej 2003a: 227)

Das abschließende Kapitel widmet Palej dem Gedächtnis, obwohl sie paradoxerweise in diesem Abschnitt viel weniger ihre russische Vergangenheit erinnert als in den vorderen Kapiteln, sondern genau gegenteilig aktuelles Geschehen hereinbrechen lässt: Es ist dies der Anschlag vom 11. September 2001 auf die Twin Towers von New York, von dem sie durch einen Anruf ihrer Kinder aus Israel erfährt. Dabei werden ihr die Augen geöffnet, ihr Blick auf Graz wird ein weiteres Mal verändert und verliert den schönen Schein, den sie ihm zuvor - zwar durchaus im Spiel mit Klischees und Stereotypen - aufgesetzt hat:

„So krass wie noch nie fiel mir plötzlich auf, dass die Grazer Realität aus nichts anderem besteht als aus Schleifen, Bändchen, Schleifen auf Bändchen, Rüschen, Pralinen, Marzipan, Blumengärten, Federn, Schachteln, Gartenzwergen ..." (Palej 2003a: 233)

So entlarvt sie ihr eigenes Grazbild zuletzt selbst als Hologramm, als Speicherbild, in dem ihre ganz subjektiven Erinnerungen manifest werden. Es wird zu einem Speicher ihres Gedächtnisses, in dem mehr festgeschrieben ist, als es ihr lieb ist; „Gedächtnislosigkeit wäre Gewähr für die Bewegung“ (Palej 2003a: 234), meint sie. 
„Im realen Graz hätten die Orte meiner Vergangenheit durchscheinen müssen [...]. Tatsächlich ist es ganz anders: Es ist Graz, das durch mein Gedächtnis durchscheint, welches mich mit einer dicken Mauer aus Gerüchen, Aromen, Farben, Geräuschen umzingelt hat und diese Festung ist fast uneinnehmbar." (Palej 2003a: 235)

In Marina Palejs semiotischer Praxis wird die De-/Konstruktion des Stadtraumes Graz verzahnt mit anderen Texträumen. Dabei werden die Schichten der sich im Laufe der Zeit überlagernden Einschreibungen dieser Texträume sichtbar, die den Charakter eines Palimpsestes haben. Trotz ihrer „nabokovesken Biographie“ (Palej 2003a: 235), wie sie ihre von Emigrations- und Migrationserfahrung geprägte Familiengeschichte bezeichnet, bleibt sie eingesperrt innerhalb der Grenzen der eigenen Erinnerungen. Diese Erkenntnis aber ist es, die ihr zuletzt zumindest einen Ausblick auf die Ent-Grenzung und eine kämpferische Geste der Be-Freiung ermöglicht:

„Vielleicht fällt mir - auf dem Schlossberg stehend - auch deshalb erst jetzt auf: dass unten, fast aus dem Zentrum der Stadt, die Freiheitsstatue herausragt. Als raffinierte Metallkonstruktion sieht sie wie eine karikaturhafte Kopie der New Yorker Statue aus. Plötzlich sehe ich, gleich wie Kafkas Held, dass sie in der Hand keine Fackel hält, sondern ein Schwert." (Palej 2003a: 235)

\section{Reflexive Nostalgie ${ }^{9}$ : Dubravka Ugrešićs „Ministarstvo Boli“}

„Mi smo barbari. Ljudi našeg plemena nose na čelima nevidljivi žig Columbove zablude. Mi putujemo zapadno i uvijek stižemo istočno, i što smo zašli zapadnija, to smo stigli istočnije, naše je pleme prokleto.“ (Ugrešić 2004: 269)

Die Texte der seit 1993 in der Migration (Berlin, USA, Amsterdam) lebenden Autorin Dubravka Ugrešić weisen zwar Gemeinsamkeiten mit der von uns erarbeiteten Migrationspoetik auf (u. a. „blurring of genre“, Dekonstruktion der traditionellen essentialistischen Konzeption von Nation, Kultur und Identität), in der thematischen Ausrichtung und Bewertung der Migrationserfahrung ist sie mit ihrem nostalgischen Erinnerungsdiskurs jedoch näher an Paradigmen der Emigrationsliteratur anzusiedeln. Obwohl sie gegen den Nationalismus in ihrer Heimat vehement auftritt und dafür von dem polemischen kroatischen Wochenblatt „Globus“ sogar als „Hexe“ und „Verräterin“ gebrandmarkt wird (vgl. Rehder 1998: 544), sieht sie auch den Gegenentwurf einer globalisierten und entgrenzten Welt, in der die postjugoslawischen Migranten weiterhin gettoisiert auf der Verliererseite leben, sehr kritisch.

Die 1949 im kroatischen Teil Jugoslawiens geborene Dubravka Ugrešić studiert ab 1968 in Zagreb Komparatistik und Russische Philologie und wird wissenschaftliche Mitarbeiterin am Institut für Literaturtheorie der Zagreber Universität. Neben ihrer wissenschaftlichen Karriere ist sie auch als Schriftstellerin erfolgreich: Sie

${ }^{9}$ Den Begriff prägt Svetlana Boym und meint damit eine Nostalgie, die den konstruktiven Charakter ihrer Objekte und die widersprüchliche Position ihres Trägers - des nostalgischen Subjekts - mit selbstironischer Distanz zu erkennen vermag (Boym 2005: 99). 
schreibt Kinderbücher, Essays und Romane und erhält internationale Auszeichnungen. 1993 verlässt sie aufgrund der politischen Situation Kroatien, wo sie auch als Schriftstellerin nicht mehr existiert (Rehder 1998: 544). ${ }^{10}$ Ugrešić verweigert eine Etikettierung als „feministische“, „postmodernistische“ und „kroatische“ Autorin und bezeichnet ihren kulturellen Hintergrund als „euro-amerikanisch“ (vgl. Rehder 1998: 544), verteidigt aber als Mitglied der ex-jugoslawischen Diaspora ihre jugoslawische Identität.

„Ministarstvo Boli““11 (2004) ist ein Roman über den Zerfall einer Identität, meint Karl-Markus Gauß in einer Rezension (2005). Die Protagonistin, Tanja Lucić, die in Zagreb mit einem serbischen Mathematiker verheiratet ist, verlässt aufgrund des zunehmenden Nationalismus Mitte der 1990er Jahre ihre Heimat. Zunächst gehen die beiden nach Berlin, ihr Mann bald darauf nach Japan. Tanja will in Europa bleiben, um dem Land, in dem sie nicht mehr leben darf und will, nahe zu sein. Sie erhält einen Lehrauftrag am kleinen Institut für Jugoslawistik der Universität Amsterdam und unterrichtet dort serbokroatische Literatur. Ein Fach mit „Ablaufdatum“, das es zukünftig so nicht mehr geben wird. Aber sie akzeptiert die Teilung des Serbokroatischen in die einzelnen „neuen“ Sprachen nicht: „Sve u svemu, ,novi“ jezici nisu me mnogo brinuli i nije mi padalo na pamet da ih razdvajam zbog razlike sadržane u nekih pedesetak riječi." (Ugrešić 2004: 45) Sie will ihre Studenten, alles Emigranten aus dem ehemaligen Jugoslawien, vor dem Identitätsverlust, mit dem der Sprachzerfall einhergeht, schützen. So gestaltet sie ihren Unterricht gänzlich unkonventionell und legt ihn als ,psychopolitisches Experiment“ (Fetz 2005: o. P.) an. Die Lehreinheiten werden zu ,jugonostalgischen“"Erinnerungsstunden, in denen sie das Projekt einer „Katalogisierung des ex-jugoslawischen Alltags“ (2004: 61) durchführen. Dabei packen alle Teilnehmer die ihnen wichtigen Erinnerungen aus ihrer verlorenen Heimat in eine für Emigranten aus Osteuropa typische rot-weiß-blau gestreifte Plastiktasche, die zur Metapher für ihren emotionalen Ballast und Proviant wird. Obwohl das Experiment zunächst gut läuft, scheinen die Studenten ihre teils traumatischen Erfahrungen dennoch lieber vergessen als erinnern zu wollen, denn Tanja wird anonym beim Institutsleiter denunziert. Völlig schockiert darüber ändert sie ihren Unterrichtsstil und macht konventionellen Literaturunterricht. Im Epilog wird ihr Leben als Emigrantin an den Rändern der westlichen Gesellschaft ${ }^{12}$ sehr resignativ als trostloses Ausharren dargestellt:

10 „Ich bin aus dem literarischen Leben Kroatiens vollkommen ausgelöscht“, sagt sie in einem Interview (cit. nach Schweiger 2006: 179)

11 Zum Titel des Romans: „Die Pointe des Titels besteht darin, dass das ,Ministerium der Schmerzen' ein ironisches Zitat von Studenten ist, die für einen holländischen ,S\&M“Versand in einer Pornoschneiderei Utensilien zusammennähen. Dieser Versand beliefert auch einen Klub, der ,Ministry of Pain' heißt. ,Schönere‘ und treffendere Titel gibt es selten." (Fetz 2005: o. P.)

12 Tanja arbeitet als Babysitterin, obwohl sie Universitätsdozentin ist. Groteskerweise lebt sie mit jenem Studenten zusammen, der sie auch denunziert hat. „Das Verhältnis der bei- 
Život je dobar prema nama. Igor obično odlazi ujutro i vraća se predvečer. [...] Jedemo polako i, začudo, govorimo malo. Naše su riječi suhe poput pijeska. Godi mi ta suhoća. Možda smo s vremenom postali Holanđani. Kažu da Holanđani govore samo onda kada imaju što za reći. (Ugrešić 2004: 301) ${ }^{13}$

Formal legt Ugrešić auch in diesem Roman, der Essay und Erzählung verwebt, die für sie kennzeichnende „ludistische Opulenz von intertextuellen Bezügen“ (Burkhart 1996: 190) vor, jene „Patchwork“- oder „Recycling“-Technik (vgl. Frangeš 1995: 540), mit der sie Fakten und Fiktionen mischt. Aufgebaut ist der Roman in fünf Teile mit anschließendem Epilog, die aus lauter kürzeren Abschnitten bestehen und das Geschehen stets aus neuer Perspektive einfangen, es aber auch mit historischen Exkursen, politischen Glossen, Gedichten und Abhandlungen zur serbischen Literatur, kroatischen Geschichte und bosnischen Mentalität erläutern (vgl. Gauß 2005: o. P.). Der Fragmentcharakter der einzelnen Abschnitte scheint den ,pprekären Erfahrungen der Exilwirklichkeit“" (Fetz 2005: o. P.) zu entsprechen.

Die ästhetischen Verfahren Ugrešićs fügen sich in das Modell der Migrationspoetik ein, wie begründen wir also unsere These von der Zuordnung der Autorin bzw. dieses Romans zum Emigrationsparadigma? Verfährt Ugrešić auf der formal-stilistischen Seite durchaus komplex und differenziert, so operiert sie auf der thematischinhaltlichen Seite mit einer ,monokausalen und monolinearen Deutung von Transition und Staatszerfall (vgl. Vidulić 2007: o. P.). Zunächst einmal steht dahinter die traumatische Kriegserfahrung, die sowohl das autobiographische als auch das fiktive Ich erlebt haben und die den Prozess der Reflexion und Narration erst in Gang setzt (vgl. Vidulić 2007: o. P.). Ugrešićs Roman ist geprägt von der Flucht vor dem Krieg, sie führt die Fragmentarisierung der Biographien von Flüchtlingen und die Instabilität von Migrationsexistenzen vor (vgl. Schweiger 2006: 178):

„Kennzeichnend für alle EmigrantInnen im Ministerium der Schmerzen, seien sie bosnischer, kroatischer oder serbischer Herkunft, ist die Erfahrung des Verlusts der eigenen Lebensgeschichte(n) in der materialisierten Form von Fotos, Büchern und den persönlichen Dingen des Lebens. Durch Migration, vor allem durch Flucht, gehen die biographischen Archive verloren oder sind nicht mehr zugänglich." (Schweiger 2006: 178)

Weiters ist es das Festhalten am Projekt des multiethnischen und multikulturellen Jugoslawiens, die sog. „Jugonostalgie“, ${ }^{14}$ die den Opferdiskurs des Textes unter-

den wird zum sadomasochistischen Albtraum, in dem das Trauma Exjugoslawien und Balkankrieg in den intimsten Innenräumen gezeigt wird.“ (Fetz 2005: o. P.)

13 Interessanterweise fehlt im Original der aussagestarke Schluss der deutschen Übersetzung des Romans über die Stimm- und Spurlosigkeit der Protagonistin und das Löschen der Erinnerung in der kroatischen Ausgabe: „Wenn meine Stimmbänder nichts mehr hergeben und meine Stirn vom Wind taub wird, verlasse ich beruhigt den Strand. Hinter mir bleibt keine Spur. Die Horizontalen der holländischen Landschaft sind wie gutes altes Löschpapier. Sie saugen alles auf ..." (Ugrešić 2005: 286)

14 Das Wort „Jugonostalgiker“ wird den Transitionsverlierern als Schimpfwort für „vaterlandslose Gesellen“ gegeben (Vidulić 2007: o. P.). 
stützt. Dazu gehört neben dem „Katalogisierungsprojekt“ beispielsweise auch der Gebrauch von Wörtern wie „Unsrige“, wie die Gruppe von Tanja sich und die Menschen aus Exjugoslawien in Differenzsetzung zu den anderen nennt, oder ,drugarica“" als Anrede für die Genossin Lehrerin. Der neuen Multikulturalität in der globalisierten Welt kann die Protagonistin nichts Positives abgewinnen, im Gegenteil, sie schildert die neue Generation von Migrant(inn) en als „,transitorische Mutanten“ sehr sarkastisch: Diese machen auf ihrem Weg in den globalisierten Kapitalismus eine Metamorphose durch und passen sich - flexibel, mobil und fluid wie sie sind - den neuen Bedingungen perfekt an. Im Gegensatz zu Entwürfen der Cultural und Postcolonial Studies, in denen Globalisierung und Migration als Gegenmodell gegen neu aufkeimende Nationalismen und Essentialismen eingeschätzt werden und die dem Schreiben von Migrant(inn)enbiographien in der Diskussion über Globalisierung und Regionalisierung und über die Auswirkungen weltweiter Migrationsbewegungen auf das Verständnis von Nation und Kultur eine positive Bedeutung zuschreiben (vgl. Schweiger 2006: 185), richtet Ugrešić ihr Augenmerk auf die Seite der Verlierer in dieser Entwicklung. Der Erfolg der einen kosmopolitischen Migranten basiert auf der Ausbeutung der anderen. Im folgenden Textausschnitt mimt sie in ironischer Weise die Sprache der globalisierten Welt, die gespickt mit Anglizismen nur mehr entlang von Schlagwörtern und inhaltslosen Phrasen verläuft:

„Iz mongolskih, rumunjskih, slovačkih, mađarskih, hrvatskih, srpskih, albanskih, bugar-
skih, bjeloruskih, moldavskih, letonskih i litvanskih sivih provincija jurnut će na evropska
i američka sveučilišta novi, kompatibilni igrači, tranzicijski mutanti, ti koji će konačno
,znati znanje‘. [...] Bit će to ljudi koji će udobno živjeti na nesreći onih kojima će poma-
gati, jer i nesreća zahtijeva management, bez managementa nesreća je tek puki neuspjeh.
[...] Bit će to mutanti, koji će, efikasni poput laboratorijskih virusa, širiti svoje mreže,
svoje networke, svoje kišobrane $i$ svoje kišobranske jedinice, svoje linkove i svoje centre.
Bit će to budući stručnjaci za televizualnu komunikaciju, za audiovizualni management,
za net i web. Bit će to planeri tuđih života i svojih karijera, koji think deeply, read wildely
and write beautifuly. Bit će to samopouzdani ljudi, ljudi multipliciranih identiteta, kozmo-
politi, globalisti, multikulturalisti, nacionalisti, predstavnici etničkih identiteta i disperziv-
nih dijasporičnih identiteta, sve u jednom, po nekoliko glava na jednim plećima, fleksibil-
ni, brzi na definiranju, samodefiniranju i redefiniranju [...] bit će to ljudi koji će u ustima
premetati riječi mobility, flexibility i fluidity kao žvakaću gumu. Bit će to mladi, progre-
sivni ljudi, plaćeni komesari European integration and enlargement procesa, radnici na
gradnji novoga poretka, specijalisti za new, unique postnational political units, za natio-
nal i postnational constellations, stručnjaci za globalization versus localization, i obratno,
advokati, koji će zdušno advokatirati ovo ili ono.“ (Ugrešić $2004: 280 \mathrm{f}$.)

Tanja Lucić verweigert zum einen den sich vollziehenden kollektiven Identitätswechsel vom jugoslawischen zum nationalistischen Projekt und verlässt aus diesem Grund ihre Heimat; obwohl sie die lokale Mythomotorik, auf die der Nationalismus baut, dekonstruiert, lehnt sie auch das Modell des globalisierten Subjekts der Migration für sich ab, sie bleibt nostalgisch ihrer Heimat treu, auch wenn sie diese als Konstrukt kritisch reflektiert. Ihre Emigration als äußere Konsequenz der Verweigerung des Nationalismus (vgl. Vidulić 2007: o. P.) führt zu Entwurzelung und Unbehaustheit. Die damit einhergehende „Freiheit des Migranten“ (Flusser 1994), deren 
Potential des Subversiven, das lineare und bruchlose Lebensentwürfe als reine Fik-

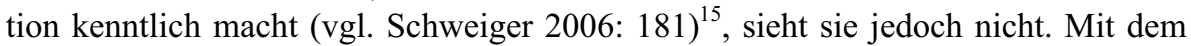
selbstgewählten Nomadentum der „postmodernen Flaneure, Spieler und Touristen“ und ihrer „Tugend der Orientierungslosigkeit“ hat ihr jugonostalgischer Entwurf nichts gemein (vgl. Vidulić 2007: o. P.). Ugrešić verharrt damit in einer „Patt-Stellung“ zwischen dekonstruktiver Kritik am nationalistischen und globalistischen System und nostalgischer Rückschau auf das multikulturelle Jugoslawien, die ihr jedoch einen utopischen Blick auf eine neue entgrenzte Welt verbaut. Insofern ist „Ministarstvo Boli““ ein literarischer Beitrag zu jener von positiver Jugoslawien-Erfahrung geprägten Erinnerungskultur, die sich der (als Terror erfahrenen) Neuordnung im Namen der (in der Verlusterfahrung verklärten) alten Ordnung verweigert (vgl. Vidulić 2007: o. P.).

\section{Abkürzungen}

Adorno 1984: $\quad$ Theodor W. Adorno, Der Essay als Form, in: Ders., Noten zur Literatur. Gesammelte Schriften Bd. II, Frankfurt a. M., 9-33

Assmann 2006: $\quad$ Aleida Assmann, Einführung in die Kulturwissenschaft. Grundbegriffe, Themen, Fragestellungen, Berlin

Benthien - Krüger-Fürhoff 1999: C. Benthien, I. Krüger-Fürhoff, Über Grenzen: Limitation und Transgression in Literatur und Ästhetik, Stuttgart - Weimar

Boym 2005: $\quad$ Svetlana Boym, Budućnost nostalgije, Beograd

Burkhart 1996: $\quad$ Dagmar Burkhart, Ludistische Texte. Zur Postmodernen Intertextualität in Dubravka Ugrešić' „Stefica cvek u raljama života“ und in „Forsiranje romana-reke“, in: D. Burkhart, V. Biti (Hrsg.), Diskurs der Schwelle: Aspekte der kroatischen Gegenwartsliteratur, Frankfurt

Fetz 2005: Bernhard Fetz, Hinter der Muttersprache, in: http://www.falter.at/ a. M. u. a., $189-203$ rezensionen $(30.11 .2006)$

Flusser 1994: $\quad$ Vilém Flusser, Von der Freiheit des Migranten. Einsprüche gegen den Nationalismus, Köln

Frangeš 1995: $\quad$ Ivo Frangeš, Geschichte der kroatischen Literatur: Von den Anfängen bis zur Gegenwart, Köln - Weimar - Wien

Gauß 2005: $\quad$ Karl-Markus Gauß, Identität: Die gestreifte Tasche, in: http://www.die presse.com (30. 11. 2006)

Gehrke 1999: Hans-Joachim Gehrke, Einleitung: Grenzgänger im Spannungsfeld von Identität und Alterität, in: M. Fludernik, H.-J. Gehrke (Hrsg.), Grenzgänger zwischen Kulturen, Würzburg, 15-24

Hannerz 1996: $\quad$ Ulf Hannerz, Transnational connections: culture, people, places, London

Hausbacher 2006: $\quad$ Eva Hausbacher, Anderswo ist überall. Ein postkolonialer Blick auf das Schreiben zwischen Ost und West anhand ausgewählter Beispiele aus der aktuellen russischen Literatur, in: E. Binder, W. Stadler, H.

15 Schweiger spricht von der „Subversivität von MigrantInnenbiographien, die nationale Identitätskonstruktionen fragwürdig erscheinen lassen und sie als kollektive Imaginationen bloßstellen, die nicht auf eine Essenz, ein Zentrum oder einen Ursprung rückführbar sind" (vgl. Schweiger 2006: 184). 
Hohnsträter 1999:

Lützeler 2005:

Palej 2003:

Palej 2003a:

Palej 2005:

Pratt 1992:

Rehder 1998:

Rösch 2004:

Schweiger 2006:

Tichomirova 2000:

Turner 1967:

Ugrešić 2004:

Ugrešić 2005:

Vidulić 2007:
Weinberger (Hrsg.), Zeit - Ort - Erinnerung. Slawistische Erkundungen aus sprach-, literatur- und kulturwissenschaftlicher Perspektive, Innsbruck, 245-259

Dirk Hohnsträter, Im Zwischenraum. Ein Lob des Grenzgängers, in: C. Benthien, I. Krüger-Fürhoff (Hrsg.), Über Grenzen. Limitation und Transgression in Literatur und Ästhetik, Stuttgart - Weimar, 231-244

Paul M. Lützeler, Postmoderne und postkoloniale deutschsprachige Literatur. Diskurs - Analyse - Kritik, Bielefeld

Marina Palej, Vychod, in: Vestnik Evropy 9 (http://magazines.russ.ru/ vestni/2003/9/palej-pr.html, 20. 08. 2007)

Marina Palej, Graz: Ein unterhaltsames Hologramm, in: K. Hoffer, A. Kolleritsch (Hrsg.), graz von aussen, Graz, 216-235

Marina Palej, V svoem jazyke ja otvečaju za každyj zvuk. Interview, in: Delo, 17. 08. 2005, Ljubljana

Mary Louise Pratt, Imperial Eyes. Trave Writing and Transculturation, London - New York

Petra Rehder, Stichwort zu Dubravka Ugrešić, in: U. Hechtfischer, R. Hof, I. Stephan, St. u. F. Veit-Wild (Hrsg.), Metzler Autorinnen Lexikon, Stuttgart, Weimar, 543-544

Heidi Rösch, Migrationsliteratur als neue Weltliteratur?, Sprachkunst XXXV,1, 89-109

Hannes Schweiger, Identitäten mit Bindestrich. Biographien von MigrantInnen, in: B. Fetz, H. Schweiger (Hrsg.), Spiegel und Maske. Konstruktionen biographischer Wahrheit, Wien, 175-188

Elena Tichomirova, Literatur der russischen Emigrant/innen, in: C. Chielino (Hrsg.), Interkulturelle Literatur in Deutschland: ein Handbuch, Stuttgart - Weimar, 166-176

Victor Turner, Betwixt and Between. The Liminal Period in , Rites de Passage', in: Ders., The Forest of Symbols. Aspects of Ndembu Ritual, Ithaca, 93-111

Dubravka Ugrešić, Ministarstvo Boli, Zagreb

Dubravka Ugrešić, Das Ministerium der Schmerzen, Berlin

Svjetlan L. Vidulić, Sonderposten im Jugoslawischen Erinnerungskrieg. Zur Exil-Prosa von Dubravka Ugrešić, in: http://www.kakanien. ac.at/beitr/fallstudie/SVidulic3.pdf (05.07. 2007)

Eva Hausbacher

Institut für Slawsitik der Universität Salzburg

Akademiestr. 24, 5020 Salzburg, Österreich

eva.hausbacher@sbg.ac.at 